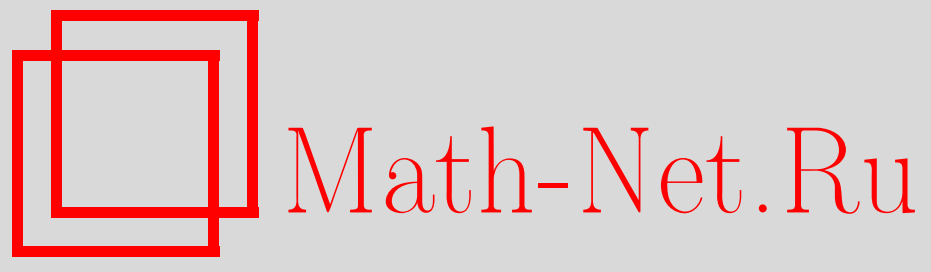

В. Нгуен-Кхак, К. Нгуен-Ван, Бесконечномерное обобщение теоремы Юнга, Матем. заметки, 2006, том 80, выпуск 2, 231-239

DOI: https://doi.org/10.4213/mzm2804

Использование Общероссийского математического портала Math-Net.Ru подразумевает, что вы прочитали и согласны с пользовательским соглашением http://www .mathnet.ru/rus/agreement

Параметры загрузки:

IP : 54.164 .48 .24

26 апреля 2023 г., 18:29:22

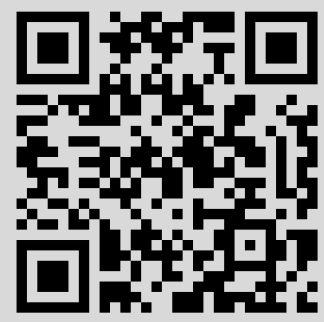




\section{БЕСКОНЕЧНОМЕРНОЕ ОБОБЩЕНИЕ ТЕОРЕМЫ ЮНГА}

\section{В. Нгуен-Кхак, К. Нгуен-Ван}

В статье дается полная характеризация экстремальных подмножеств в гильбертовых пространствах, что является бесконечномерным обобщением классической теоремы Юнга. Мы исследуем также поведение совокупности точек, близких к сфере Чебышева для таких подмножеств относительно мер некомпактности Куратовского и Хаусдорфа.

Библиография: 9 названий.

1. Введение. Пусть $(X,\|\cdot\|)$ - некоторое банахово пространство. Для непустого ограниченного подмножества $A$ в $X$ и непустого подмножества $B$ в $X$ мы будем использовать следующие обозначения: $d(A):=\sup \{\|x-y\|: x, y \in A\}$ - диаметр $A$;

$$
r_{B}(A):=\inf _{y \in B} \sup _{x \in A}\|x-y\|
$$

- относительный радиус Чебышева подмножества $A$ по отношению к $B$, в частности, $r(A):=r_{\overline{\mathrm{co}} A}(A)$, где $\overline{\mathrm{co}} A$ обозначает замыкание выпуклой оболочки подмножества $A$;

$$
C_{B}(A):=\left\{y \in B: \sup _{x \in A}\|x-y\|=r_{B}(A)\right\}
$$

- множество чебышевских центров подмножества $A$ в $B$.

Константа Юнга пространства $X$ определяется как

$$
J(X):=\sup \left\{r_{X}(A): A \subset X \text { c } d(A)=1\right\} .
$$

В связи с изучением равномерно-нормальной структуры рассматривается также другая важная геометрическая константа - относительная константа Юнга пространства $X$ (см. [1]):

$$
J_{s}(X):=\sup \{r(A): A \subset X, \quad \mathrm{c} d(A)=1\} .
$$

В случае, когда $X$ - гильбертово пространство, известно, что $C_{X}(A)$ состоит из единственной точки, которая принадлежит замыканию выпуклой оболочки $\overline{\mathrm{co}} A$ подмножества $A$ (см. [2]). Поэтому в этом случае $J(X)=J_{s}(X)$. Классическая теорема Юнга утверждает, что для $n$-мерного евклидова пространства $X=E^{n}$ мы имеем

$$
J\left(E^{n}\right)=J_{s}\left(E^{n}\right)=\sqrt{\frac{n}{2(n+1)}}
$$

(C) В. НГУЕН-КХАК, К. НГУЕН-ВАН, 2006 
[3], [4]. Константа Юнга $J(H)$ гильбертова пространства (бесконечномерный случай) была вычислена позже в [5] (ср. [6], [1], [7]):

$$
J(H)=J_{s}(H)=\frac{1}{\sqrt{2}} .
$$

ОПРедЕЛЕНИЕ. Мы говорим, что ограниченное подмножество $A$ в $X$, состоящее из более чем одной точки, является экстремальным (соответственно относительноэкстремалъным), если $r_{X}(A)=J(X) d(A)$ (соответственно $r(A)=J_{s}(X) d(A)$ ).

Заметим, что если $X$ - гильбертово пространство, то два этих понятия совпадают, так что в этом случае мы будем говорить просто об экстремальных подмножествах. Из второй части упомянутой выше теоремы Юнга следует, что ограниченное подмножество $A$ в $E^{n}$ является экстремальным, если и только если $A$ содержит правильный $n$-симплекс с ребрами длины $d(A)$. В бесконечномерном случае $(X=H)$ частичный результат был получен Гулевичем [8]: если $A$ - относительно компактное подмножество в гильбертовом пространстве с $d(A)>0$, то

$$
r(A)<\frac{1}{\sqrt{2}} d(A) .
$$

Другими словами, если $A$ - экстремальное подмножество в гильбертовом пространстве, то $A$ не является относительно компактным.

Наша цель в этой статье - дать полную характеризацию экстремальных подмножеств в гильбертовых пространствах, что является бесконечномерным обобщением второй части классической теоремы Юнга.

ГЛАВНАЯ ТЕОРЕмА. Пусть $A$ - некоторое экстремальное подмножество в гильбертовом пространстве $H$ c $d(A)=\sqrt{2}$. Тогда $\chi(A)=1$. Более того, при каждом $\varepsilon \in(0, \sqrt{2})$ для каждого положительного целого р существует некоторый р-симплекс $\Delta(\varepsilon, p)$ с вершинами в $A$ такой, что длина каждого ребра симплекса $\Delta(\varepsilon, p)$ не меньше, чем $\sqrt{2}-\varepsilon$.

Обратно, если $d(A)=\sqrt{2}$ и для каждого $\varepsilon \in(0, \sqrt{2})$ при каждом положительном иелом $p$ существует некоторый $p$-симплекс $\Delta(\varepsilon, p)$ с вериинами в $A$, ребра которого имеют длину не меньше, чем $\sqrt{2}-\varepsilon$, то $A$ - экстремальное подмножество.

В формулировке главной теоремы $\chi(A)$ обозначает меру некомпактности Хаусдорфа подмножества $A$, т.е. нижняя грань таких положительных $r$, что $A$ может быть покрыто конечным числом шаров с центрами в $H$ и радиуса $r$. Кроме того, основываясь на одном наблюдении из [7] (“лемма о грибах"), мы доказываем также результат, касающийся поведения совокупности точек, близких к сфере Чебышева, для таких подмножеств относительно меры некомпактности. Этот результат означает, что основной вклад в меру некомпактности происходит именно от этой части данного экстремального подмножества.

\section{2. Меры некомпактности экстремальных подмножеств.}

Теорема 1. Пусть $A$ - некоторое экстремальное подмножество в гилъбертовом пространстве $H$ с $r(A)=1$. Тогда мы имеем $\alpha(A)=\sqrt{2}$. 
Здесь $\alpha(A)$ обозначает меру некомпактности Куратовского, которая по определению есть нижная грань таких положительных $d$, что $A$ может быть покрыто конечным числом подмножеств диаметра $d$.

ПЕРВОЕ ДОКАЗАТЕЛЬСТВО. Из условия $r(A)=1$ следует, что для каждого целого числа $n \geqslant 2$ мы имеем

$$
\bigcap_{x \in A} B\left(x, 1-\frac{1}{n}\right)=\varnothing,
$$

где $B(x, r)$ обозначает замкнутый шар с центром в $x$ и радиусом $r$, который слабо компактен, поскольку $H$ рефлексивно. Поэтому в $A$ существуют

$$
x_{p_{n-1}+1}, \quad x_{p_{n-1}+2}, \quad \ldots, \quad x_{p_{n}}
$$

такие, что

$$
\bigcap_{i=p_{n-1}+1}^{p_{n}} B\left(x_{i}, 1-\frac{1}{n}\right)=\varnothing
$$

(с соглашением $\left.p_{1}=0\right)$.

Полагая

$$
A_{n}:=\left\{x_{p_{n-1}+1}, x_{p_{n-1}+2}, \ldots, x_{p_{n}}\right\},
$$

мы обозначаем через $c_{n}$ чебышевский центр подмножества $A_{n}$ в $H$, и пусть $r_{n}:=$ $r\left(A_{n}\right)$, тогда $r_{n}>1-1 / n$.

Пусть $S(c, r)$ обозначает сферу с центром $c$ и радиусом $r$. Из доказательства классической теоремы Юнга мы знаем, что $A \cap S\left(c_{n}, r_{n}\right) \neq \varnothing$ и $c_{n} \in \operatorname{co}\left(A_{n} \cap S\left(c_{n}, r_{n}\right)\right)$. Так что существуют $y_{q_{n-1}+1}, y_{q_{n-1}+2}, \ldots, y_{q_{n}}$ в $A_{n} \cap S\left(c_{n}, r_{n}\right)$ (с соглашением $q_{1}=0$ ) и положительные числа $t_{q_{n-1}+1}, t_{q_{n-1}+2}, \ldots, t_{q_{n}}$ такие, что

$$
c_{n}=\sum_{q_{n-1}<i \leqslant q_{n}} t_{i} y_{i}, \quad \sum_{q_{n-1}<i \leqslant q_{n}} t_{i}=1 .
$$

Мы утверждаем, что

$$
\alpha\left(\left\{y_{q_{n-1}+1}, y_{q_{n-1}+2}, \ldots, y_{q_{n}}\right\}_{n=2}^{\infty}\right)=\sqrt{2} .
$$

Предположим "от противного", что $\alpha\left(\left\{y_{q_{n-1}+1}, y_{q_{n-1}+2}, \ldots, y_{q_{n}}\right\}_{n=2}^{\infty}\right)<\sqrt{2}$. Тогда мы можем выбрать такое $\varepsilon_{0} \in(0, \sqrt{2})$, что $\alpha\left(\left\{y_{q_{n-1}+1}, y_{q_{n-1}+2}, \ldots, y_{q_{n}}\right\}_{n=2}^{\infty}\right) \leqslant \sqrt{2}-\varepsilon_{0}$, и, тем самым, подмножества $D_{1}, D_{2}, \ldots, D_{m}$ в $H$ с условием

$$
d\left(D_{i}\right) \leqslant \sqrt{2}-\varepsilon_{0}, \quad i=1,2, \ldots, m
$$

такие, что

$$
\left\{y_{q_{n-1}+1}, y_{q_{n-1}+2}, \ldots, y_{q_{n}}\right\}_{n=2}^{\infty} \subset \bigcup_{i=1}^{m} D_{i} .
$$

Существует по крайней мере одно подмножество среди $D_{1}, D_{2}, \ldots, D_{m}$, скажем, $D_{1}$, удовлетворяющее условию, что найдется бесконечно много $n$ таких, что

$$
\sum_{i \in J_{n}} t_{i} \geqslant \frac{1}{m}
$$


где

$$
J_{n}:=\left\{i \in\left[q_{n-1}+1, q_{n}\right]: y_{i} \in D_{1}\right\} .
$$

Для каждого $n$, удовлетворяющего (1), и фиксированного $j \in J_{n}$ мы имеем

$$
\begin{aligned}
\sum_{q_{n-1}<i \leqslant q_{n}} t_{i}\left\|y_{i}-y_{j}\right\|^{2} & =\sum_{q_{n-1}<i \leqslant q_{n}} t_{i}\left\|y_{i}-c_{n}+c_{n}-y_{j}\right\|^{2} \\
& =\sum_{q_{n-1}<i \leqslant q_{n}} t_{i}\left(\left\|y_{i}-c_{n}\right\|^{2}+\left\|y_{j}-c_{n}\right\|^{2}-2\left(y_{i}-c_{n}, y_{j}-c_{n}\right)\right) \\
& =2 r_{n}^{2}-2\left(\sum_{q_{n-1}<i \leqslant q_{n}} t_{i} y_{i}-c_{n}, y_{j}-c_{n}\right) \\
& =2 r_{n}^{2}>2\left(1-\frac{1}{n}\right)^{2}>2-\frac{4}{n},
\end{aligned}
$$

где $(\cdot, \cdot)$ обозначает скалярное произведение в $H$.

С другой стороны,

$$
\begin{aligned}
\sum_{q_{n-1}<i \leqslant q_{n}} t_{i}\left\|y_{i}-y_{j}\right\|^{2} & =\sum_{i \in J_{n}} t_{i}\left\|y_{i}-y_{j}\right\|^{2}+\sum_{q_{n-1}<i \leqslant q_{n}, i \notin J_{n}} t_{i}\left\|y_{i}-y_{j}\right\|^{2} \\
& \leqslant\left(\sqrt{2}-\varepsilon_{0}\right)^{2} \sum_{i \in J_{n}} t_{i}+2\left(1-\sum_{i \in J_{n}} t_{i}\right) \\
& =2-\left[2-\left(2-\varepsilon_{0}\right)^{2}\right]\left(\sum_{i \in J_{n}} t_{i}\right) \leqslant 2-\left[2-\left(2-\varepsilon_{0}\right)^{2}\right] \frac{1}{m}
\end{aligned}
$$

Поэтому

$$
2-\left[2-\left(2-\varepsilon_{0}\right)^{2}\right] \frac{1}{m}>2-\frac{4}{n}
$$

при фиксированных $\varepsilon_{0}, m$ и для всех $n$, удовлетворяющих $(1),-$ противоречие.

Следовательно $\alpha\left(\left\{y_{q_{n-1}+1}, y_{q_{n-1}+2}, \ldots, y_{q_{n}}\right\}_{n=2}^{\infty}\right)=\sqrt{2}$. А так как $d(A)=\sqrt{2}$, мы заключаем, что $\alpha(A)=\sqrt{2}$.

Для второго доказательства нам понадобится следующая лемма, которая является вариацией леммы 4 (“леммы о грибах") из [7].

Лемма 2. Пусть $A$ - непустое ограниченное подмножество гилъбертова пространства $H$; г и с-соответственно чебышевский радиус подмножества $A$ относительно $H$ и чебышевский центр подмножества $A$ в $H$. Тогда $c \in \overline{\mathrm{co}} A_{\varepsilon} u$ $r=r\left(A_{\varepsilon}\right)$ для любого $\varepsilon \in(0, r)$, где $A_{\varepsilon}:=A \backslash B(c, r-\varepsilon)$.

ДокАЗАТЕЛЬСтво ЛЕммы 2. Предположим "противное", что $c$ не является чебышевским центром $A_{\varepsilon}$ в $H$, тогда $r_{1}:=r\left(A_{\varepsilon}\right)<r$. Обозначая через $c_{1}$ чебышевский центр $A_{\varepsilon}$ в $H$, мы выбираем $c^{\prime}=\alpha c_{1}+(1-\alpha) c$ для некоторого $\alpha \in(0,1)$ такого, что $0<\left\|c-c^{\prime}\right\|<\varepsilon$.

Возьмем некоторую точку $x \in A$. Если $x \in A_{\varepsilon}$, то

$$
\left\|x-c^{\prime}\right\| \leqslant \alpha\left\|x-c_{1}\right\|+(1-\alpha)\|x-c\| \leqslant \alpha r_{1}+(1-\alpha) r<r .
$$

А если $x \in A \backslash A_{\varepsilon}$, то

$$
\left\|x-c^{\prime}\right\| \leqslant\|x-c\|+\left\|c-c^{\prime}\right\|<r-\varepsilon+\left\|c-c^{\prime}\right\|<r .
$$


В любом случае мы имеем $A \subset B\left(c^{\prime}, r^{\prime}\right)$ с $r^{\prime}<r$, противоречие. Лемма доказана.

ВТОРОЕ ДОКАЗАТЕЛЬСТвО ТЕОРЕМЫ 1 . Ввиду леммы 2, беря $\varepsilon=1 / n$ для каждого целого $n \geqslant 2$, мы получаем: $c \in \overline{\mathrm{co}}(A \backslash B(c, 1-1 / n))$. Поэтому существуют $x_{p_{n-1}+1}, x_{p_{n-1}+2}, \ldots, x_{p_{n}}$ в $A \backslash B(c, 1-1 / n)$ и положительные числа

$$
t_{p_{n-1}+1}, \quad t_{p_{n-1}+2}, \quad \ldots, \quad t_{p_{n}}
$$

(с соглашением $p_{1}=0$ ) такие, что

$$
\sum_{p_{n-1}<i \leqslant p_{n}} t_{i}=1, \quad\left\|\sum_{p_{n-1}<i \leqslant p_{n}} t_{i} x_{i}-c\right\|<\frac{1}{n} .
$$

Мы покажем, что

$$
\alpha\left(\left\{x_{p_{n-1}+1}, x_{p_{n-1}+2}, \ldots, x_{p_{n}}\right\}_{n=2}^{\infty}\right)=\sqrt{2} .
$$

Снова будем вести доказательство от противного, предположив, что $\alpha\left(\left\{x_{p_{n-1}+1}\right.\right.$, $\left.\left.x_{p_{n-1}+2}, \ldots, x_{p_{n}}\right\}_{n=2}^{\infty}\right)<\sqrt{2}$. Тогда найдется такое $\varepsilon_{0} \in(0, \sqrt{2})$, что $\alpha\left(\left\{x_{p_{n-1}+1}\right.\right.$, $\left.\left.x_{p_{n-1}+2}, \ldots, x_{p_{n}}\right\}_{n=2}^{\infty}\right) \leqslant \sqrt{2}-\varepsilon_{0}$, и, тем самым, существуют такие подмножества $D_{1}, D_{2}, \ldots, D_{m}$ в $H$ с условием $d\left(D_{i}\right) \leqslant \sqrt{2}-\varepsilon_{0}, i=1,2, \ldots, m$, что

$$
\left\{x_{p_{n-1}+1}, x_{p_{n-1}+2}, \ldots, x_{p_{n}}\right\}_{n=2}^{\infty} \subset \bigcup_{i=1}^{m} D_{i} .
$$

Как и в первом доказательстве, мы можем найти среди $D_{1}, D_{2}, \ldots, D_{m}$ подмножество, скажем $D_{1}$, такое, что имеется бесконечно много $n$, удовлетворяющих условию

$$
\sum_{i \in I_{n}} t_{i} \geqslant \frac{1}{m}
$$

где

$$
I_{n}:=\left\{i \in\left[p_{n-1}+1, p_{n}\right]: x_{i} \in D_{1}\right\} .
$$

Аналогично для каждого $n$, удовлетворяющего (2), и фиксированного $j \in I_{n}$ мы имеем

$$
\begin{aligned}
\sum_{p_{n-1}<i \leqslant p_{n}} t_{i}\left\|x_{i}-x_{j}\right\|^{2} & =\sum_{p_{n-1}<i \leqslant p_{n}} t_{i}\left\|x_{i}-c+c-x_{j}\right\|^{2} \\
& =\sum_{p_{n-1}<i \leqslant p_{n}} t_{i}\left(\left\|x_{i}-c\right\|^{2}+\left\|x_{j}-c\right\|^{2}-2\left(x_{i}-c, x_{j}-c\right)\right) \\
& >2\left(1-\frac{1}{n}\right)^{2}-2\left(\sum_{p_{n-1}<i \leqslant p_{n}} t_{i} x_{i}-c, x_{j}-c\right) \\
& \geqslant\left(1-\frac{1}{n}\right)^{2}-2 \frac{1}{n}>2-\frac{6}{n},
\end{aligned}
$$

а также

$$
\sum_{p_{n-1}<i \leqslant p_{n}} t_{i}\left\|x_{i}-x_{j}\right\|^{2} \leqslant 2-\left[2-\left(\sqrt{2}-\varepsilon_{0}\right)^{2}\right] \frac{1}{m} .
$$


Поэтому

$$
2-\left[2-\left(2-\varepsilon_{0}\right)^{2}\right] \frac{1}{m}>2-\frac{6}{n}
$$

для всех $n$, удовлетворяющих (2), - противоречие.

Таким образом, $\alpha\left(\left\{x_{p_{n-1}+1}, x_{p_{n-1}+2}, \ldots, x_{p_{n}}\right\}_{n=2}^{\infty}\right)=\sqrt{2}$, откуда и следует $\alpha(A)=$ $\sqrt{2}$.

Как непосредственное следствие мы получаем упомянутый во введении результат Гулевича.

СЛЕДСТВИЕ [8]. Пусть $A$ - относительно компактное подмножество в гильбертовом пространстве $H$ c $d(A)>0$. Тогда $r(A)<(1 / \sqrt{2}) d(A)$.

ЗАмечАние 1. В [7] дано другое доказательство равенства $J(H)=J_{s}(H)=$ $1 / \sqrt{2}$, по существу принадлежащее Штейнлейну. Его сущность лежит несколько глубже, а именно, в следующем интересном неравенстве между характеристикой Лифшица $\varkappa_{0}(X)$ и относительной константой Юнга $J_{s}(X)$ банахова пространства $X$ : $\varkappa_{0}(X) \leqslant\left(J_{s}(X)\right)^{-1}$ (ср. [9]). Отметим только, что это неравенство может быть обобщено на метрические пространства со структурой выпуклости. Мы вернемся к этой теме в последующей публикации.

ЗАмеЧАниЕ 2. С помощью леммы 2 мы видим, что $A_{\varepsilon}$ также является экстремальным подмножеством и $\alpha\left(A_{\varepsilon}\right)=\sqrt{2}$ при $\varepsilon \in(0,1)$.

ЗАмечАниЕ 3 . Хотя $\alpha\left(A_{\varepsilon}\right)=\sqrt{2}$ при $\varepsilon \in(0,1)$, но может случиться, что $\overline{\operatorname{co}} A \cap$ $S(c, 1)=\varnothing$ (ср. [7]). Возникает вопрос: что можно сказать о $\alpha(A \cap S(c, 1))$, когда $A \cap S(c, 1) \neq \varnothing$ ? Ответ таков: $\alpha(A \cap S(c, 1))$ может принимать любое значение из $[0, \sqrt{2}]$. Ниже мы приводим примеры.

ПримеР 1. Пусть $\left\{e_{n}\right\}_{n=1}^{\infty}-$ бесконечная последовательность ортонормированных векторов в гильбертовом пространстве $H$. Положим

$$
A_{1}:=\left\{\left(1-\frac{1}{n}\right) e_{n}\right\}_{n=1}^{\infty}
$$

и $A_{2}:=\left\{x_{1}, x_{2}, \ldots, x_{n}, \ldots\right\} \mathrm{c}$

$$
\begin{aligned}
& x_{1}:=\frac{1}{\sqrt{2}} e_{1}+\frac{1}{\sqrt{2}} e_{2}, \quad x_{2}:=\frac{1}{\sqrt{2}} e_{1}+\frac{1}{2} e_{2}+\frac{1}{2} e_{3}, \\
& x_{n}:=\frac{1}{\sqrt{2}} e_{1}+\frac{1}{\sqrt{2^{2}}} e_{2}+\cdots+\frac{1}{\sqrt{2^{n}}} e_{n}+\frac{1}{\sqrt{2^{n}}} e_{n+1}, \quad \ldots .
\end{aligned}
$$

Легко видеть, что $r\left(A_{1}\right)=1, d\left(A_{1}\right)=\sqrt{2}$ и 0 является чебышевским центром подмножества $A_{1}$ в $H$. Далее, $\left\|x_{n}\right\|=1$ для каждого $n$,

$$
\begin{gathered}
\left\|x_{m}-\left(1-\frac{1}{n}\right) e_{n}\right\| \leqslant \sqrt{2} \quad \forall m, n, \\
\left\|x_{n+p}-x_{n}\right\|^{2}=\frac{1}{2^{n}} \rightarrow 0 \quad \text { при } \quad n \rightarrow \infty .
\end{gathered}
$$

Следовательно, $\left\{x_{n}\right\}$ является последовательностью Коши, и мы получаем $\alpha\left(A_{2}\right)=0$. 
Теперь для множества $A:=A_{1} \cup A_{2}$ мы имеем $r(A)=1, d(A)=\sqrt{2}$ и 0 является чебышевским центром подмножества $A$ в $H$. Очевидно,

$$
A \cap S(0,1)=\overline{\mathrm{co}} A \cap S(0,1)=A_{2} .
$$

ПримеР 2. Пусть $\left\{e_{n}\right\}_{n=1}^{\infty}$ и $A_{1}$ такие же, как в примере 1. Для каждого $\gamma \in$ $(0, \sqrt{2}]$ с $\beta:=\gamma / \sqrt{2} \in(0,1]$ мы выбираем $\lambda \in[0,1)$ из формулы $\lambda^{2}+\beta^{2}=1$. Обозначим через $A_{2}:=\left\{y_{1}, y_{2}, \ldots, y_{n}, \ldots\right\}$ с

$$
y_{1}:=\lambda e_{1}+\beta e_{2}, \quad y_{2}:=\lambda e_{1}+\beta e_{3}, \quad \ldots, \quad y_{n}:=\lambda e_{1}+\beta e_{n+1}, \quad \ldots
$$

Очевидно, $\left\|y_{n}\right\|=1$ для каждого $n,\left\|y_{n}-y_{m}\right\|=\sqrt{2} \beta=\gamma$ для всех $m \neq n$. Полагая $A:=A_{1} \cap A_{2}$, мы получаем $r(A)=1, d(A)=\sqrt{2}, 0$ является чебышевским центром подмножества $A$ в $H$ и $A \cap S(0,1)=\overline{\operatorname{co}} A \cap S(0,1)=A_{2}$ с $\alpha\left(A_{2}\right)=\gamma$.

3. Доказательство главной теоремы. Из первого доказательства теоремы 1 мы построили последовательность $y_{q_{n-1}+1}, y_{q_{n-1}+2}, \ldots, y_{q_{n}}$ в $A_{n} \cap S\left(c_{n}, r_{n}\right)$ (с $\left.q_{1}=0\right)$ и положительные $t_{q_{n-1}+1}, t_{q_{n-1}+2}, \ldots, t_{q_{n}}$ для каждого целого $n \geqslant 2$, удовлетворяющего условиям

$$
c_{n}=\sum_{q_{n-1}<i \leqslant q_{n}} t_{i} y_{i}, \quad \sum_{q_{n-1}<i \leqslant q_{n}} t_{i}=1 .
$$

Мы утверждаем, что

$$
\chi\left(\left\{y_{q_{n-1}+1}, y_{q_{n-1}+2}, \ldots, y_{q_{n}}\right\}_{n=2}^{\infty}\right)=1 .
$$

Для доказательства этого предположим, что $A$ может быть покрыто конечным числом шаров радиуса $r: B_{1}, B_{2}, \ldots, B_{m}$. Тогда среди $B_{1}, B_{2}, \ldots, B_{m}$ найдется такой шар, скажем $B_{1}$, что имеется бесконечно много $n$, удовлетворяющих неравенству

$$
\sum_{i \in J_{n}} t_{i} \geqslant \frac{1}{m}
$$

где

$$
J_{n}:=\left\{i \in\left[q_{n-1}+1, q_{n}\right]: y_{i} \in B_{1}\right\} .
$$

Как в доказательстве теоремы 1 , мы имеем

$$
\sum_{q_{n-1}<i \leqslant q_{n}} t_{i}\left\|y_{i}-y_{j}\right\|^{2}=2 r_{n}^{2}>2-\frac{4}{n}
$$

для каждого фиксированного $j \in\left[q_{n-1}+1, q_{n}\right]$.

Из (4) следует оценка

$$
\sum_{i \in I_{n j}} t_{i}<\frac{1}{\sqrt{n}}
$$

где

$$
\begin{gathered}
I_{n j}:=\left\{i \in\left[q_{n-1}+1, q_{n}\right]:\left\|y_{i}-y_{j}\right\|^{2}<2-\frac{4}{\sqrt{n}}\right\}, \quad j \in\left[q_{n-1}+1, q_{n}\right], \\
2\left(1-t_{j}\right) \geqslant \sum_{q_{n-1}<i \leqslant q_{n}} t_{i}\left\|y_{i}-y_{j}\right\|^{2}=2 r_{n}^{2}>2-\frac{4}{n} .
\end{gathered}
$$


Это влечет за собой оценку $t_{j}<2 / n$ для каждого $j \in\left[q_{n-1}+1, q_{n}\right]$. Поэтому если $n$ удовлетворяет (3), то $\left|J_{n}\right|(2 / n)>1 / m$, или, что эквивалентно, $\left|J_{n}\right|>n /(2 m)$ (здесь $\left|J_{n}\right|$ означает мощность множества $J_{n}$ ).

Для каждого $n$, удовлетворяющего $(3)$, и $j \in J_{n}$ обозначим

$$
\begin{gathered}
J_{n}\left(y_{j}\right):=\left\{i \in J_{n}:\left\|y_{i}-y_{j}\right\|^{2} \geqslant 2-\frac{4}{\sqrt{n}}\right\}, \\
\hat{J}_{n}\left(y_{j}\right):=\left\{y_{i}: i \in J_{n}\left(y_{j}\right)\right\} .
\end{gathered}
$$

Очевидно, из (5) мы получаем

$$
\begin{gathered}
\sum_{i \in J_{n} \backslash J_{n}\left(y_{j}\right)} t_{i}<\frac{1}{\sqrt{n}}, \\
\sum_{i \in J_{n}\left(y_{j}\right)} t_{i}>\frac{1}{m}-\frac{1}{\sqrt{n}} .
\end{gathered}
$$

Для каждого положительного целого $p$ выбираем $n$, удовлетворяющее (3) и достаточно большое, чтобы выполнялось неравенство $(p+1) / \sqrt{n} \leqslant 1 / \sqrt{m}$. Мы утверждаем, что для любого выбора $i_{1}, i_{2}, \ldots, i_{p} \in J_{n}$ мы имеем

$$
\bigcap_{k=1}^{p} J_{n}\left(y_{i_{k}}\right) \neq \varnothing
$$

В самом деле, иначе $\bigcap_{k=1}^{p} J_{n}\left(y_{i_{k}}\right)=\varnothing$ могло бы повлечь за собой

$$
J_{n}\left(y_{i_{1}}\right) \subset J_{n} \backslash\left(\bigcap_{k=2}^{p} J_{n}\left(y_{i_{k}}\right)\right)=\bigcup_{k=2}^{p}\left(J_{n} \backslash J_{n}\left(y_{i_{k}}\right)\right) .
$$

Следовательно, ввиду (6) и (7)

$$
\frac{1}{m}-\frac{1}{\sqrt{n}}<\sum_{\alpha \in J_{n}\left(y_{i_{1}}\right)} t_{\alpha} \leqslant \sum_{k=2}^{p} \sum_{\alpha \in J_{n} \backslash J_{n}\left(y_{i_{k}}\right)} t_{\alpha}<(p-1) \frac{1}{\sqrt{n}} .
$$

Таким образом, $1 / m<p / \sqrt{n}$, и это противоречило бы выбору $n$ и $p$.

Далее, из (8) следует, что если $1 \leqslant k \leqslant p$ и $i_{1}, i_{2}, \ldots, i_{k} \in J_{n}$, то $\bigcap_{\alpha=1}^{k} \hat{J}_{n}\left(y_{i_{\alpha}}\right) \neq \varnothing$. $\mathrm{C} n$ и $p$ такими, как выше, зафиксируем $j \in J_{n}$. Положив $z_{1}:=y_{j}$, мы построим последовательно

$$
z_{2} \in \hat{J}_{n}\left(z_{1}\right), \quad z_{3} \in \hat{J}_{n}\left(z_{1}\right) \cap \hat{J}_{n}\left(z_{2}\right), \quad \ldots, \quad z_{p+1} \in \bigcap_{i=1}^{p} \hat{J}_{n}\left(z_{i}\right) .
$$

Очевидно, $\left\|z_{i}-z_{j}\right\|^{2} \geqslant 2-4 / \sqrt{n}$ для всех $i \neq j$ в $\{1,2, \ldots, p+1\}$. Теперь для заданного $\varepsilon \in(0, \sqrt{2})$ выберем $n$ таким же, как и выше, и к тому же достаточно большим, чтобы $2-4 / \sqrt{n} \geqslant(\sqrt{2}-\varepsilon)^{2}$. Мы видим, что $z_{1}, z_{2}, \ldots, z_{p+1}$ образуют $p$-симплекс $\Delta(\varepsilon, p)$, ребра которого имеют длину не меньше, чем $\sqrt{2}-\varepsilon$.

Мы покажем теперь, что $r \geqslant 1\left(r\right.$ - радиус шаров $\left.B_{1}, B_{2}, \ldots, B_{m}\right)$. Пусть $c^{\prime}$ и $r^{\prime}$ соответственно обозначают чебышевский центр симплекса $\Delta(\varepsilon, p)$ в $H$ и его 
чебышевский радиус относительно $H$. Из доказательства классической теоремы Юнга следует, что существуют неотрицательные $\alpha_{1}, \alpha_{2}, \ldots, \alpha_{p+1}$ с $\sum_{i=1}^{p+1} \alpha_{i}=1$ и $c^{\prime}=\sum_{i=1}^{p+1} \alpha_{i} z_{i}$. Далее, для каждого $j \in\{1,2, \ldots, p+1\}$

$$
\begin{aligned}
\left(2-\frac{4}{\sqrt{n}}\right)\left(1-\alpha_{j}\right) & \leqslant \sum_{i=1}^{p+1} \alpha_{i}\left\|z_{i}-z_{j}\right\|^{2}=\sum_{i=1}^{p+1} \alpha_{i}\left\|z_{i}-c^{\prime}+c^{\prime}-z_{j}\right\|^{2} \\
& =\sum_{i=1}^{p+1} \alpha_{i}\left(\left\|z_{i}-c^{\prime}\right\|^{2}+\left\|z_{j}-c^{\prime}\right\|^{2}\right)-\left(\sum_{i=1}^{p+1} \alpha_{i}\left(z_{i}-c^{\prime}\right), z_{j}-c^{\prime}\right) \\
& \leqslant 2\left(p^{\prime}\right)^{2} .
\end{aligned}
$$

Таким образом,

$$
\left(2-\frac{4}{\sqrt{n}}\right) \sum_{j=1}^{p+1}\left(1-\alpha_{j}\right) \leqslant 2(p+1)\left(r^{\prime}\right)^{2},
$$

или, что эквивалентно,

$$
r^{\prime} \geqslant \sqrt{\frac{(2-4 / \sqrt{n}) p}{2(p+1)}} .
$$

Правая часть (9) стремится к 1 при $p \rightarrow \infty$. Очевидно, $r \geqslant r^{\prime}$, поскольку $\Delta(\varepsilon, p) \subset$ $B_{1}$. Это влечет за собой $r \geqslant 1$, как утверждалось. Мы заключаем отсюда, что $\chi(A)=1$.

Обратно, если $d(A)=\sqrt{2}$, для каждого $\varepsilon \in(0, \sqrt{2})$ и каждого положительного целого $p \quad A$ содержит $p$-симплекс $\Delta(\varepsilon, p)$ с ребрами длины $\geqslant \sqrt{2}-\varepsilon$, то мы видим непосредственно, что $A$ является экстремальным подмножеством.

\section{СПИСОК ЦИТИРОВАННОЙ ЛИТЕРАТУРЫ}

[1] W. L. Bynum, "Normal structure coefficients for Banach spaces", Pacific J. Math., 86 (1980), 427-436.

[2] А. Л. Гаркави, "О чебышевском центре и выпуклой оболочке множества", УМН, 19:6 (1964), 139-145.

[3] H. W.E. Jung, "Über die kleinste Kugel, die eine räumliche Figur einschliesst", J. Reine Angew. Math., 123 (1901), 241-257.

[4] Л. Данцер, Б. Грюнбаум, В. Кли, Теорема Хелли, Мир, М., 1968.

[5] N. A. Routledge, "A result in Hilbert space", Quart. J. Math., Oxford, 3:9 (1952), 12-18.

[6] В.И. Бердышев, "Связь между неравенством Джексона и одной геометрической задачей", Матем. заметки, 3:3 (1968), 327-338.

[7] J. Daneš, "On the radius of a set in a Hilbert space", Comm. Math. Univ. Carolina, 25:2 (1984), 355-362.

[8] Н. М. Гулевич, "Радиус компактного множества в гильбертовом пространстве", Записки научных семинаров ЛОМИ, 164 (1988), 157-158.

[9] J.R.L. Webb, W. Zhao, "On connections between set and ball measures of noncompactness", Bull. London Math. Soc., 22 (1990), 471-477.

\section{В. Нгуен-Кхак}

Поступило

Институт математики Вьетнама, г. Ханой

07.06.2005

E-mail: nkviet@math.ac.vn

\section{К. Нгуен-Ван}

Ханойский педагогический институт 